\title{
Metabolomics approach to tap the potential of essential oils against Madurella mycetomatis- One of the most neglected fungal infections
}

Abd Algaffar SO1, Veres Katalin², Hohmann Judit², Elamin Mubarak¹, van de Sande WWJ ${ }^{3}$, and Khalid SA ${ }^{1}$.

${ }^{1}$ Faculty of Pharmacy, University of Science \& Technology, ${ }^{2}$ Faculty of Pharmacy, University of Szeged, Szeged, Hungary,

${ }^{3}$ Erasmus MC, Department of Medical Microbiology and Infectious Diseases, Rotterdam, The Netherlands

\section{Objectives}

There is an increasing demand to develop antifungals to combat Madurella mycetomatis which has been recently added to the list of Neglected Tropical Diseases (NTDs) by the 69th World Health Assembly in May 2016 as one of the most neglected and aggressive fungal infections. Despite the number of the knowledge gaps associated with $M$. mycetomatis ${ }^{1}$ the treatment option remains the most challenging due to the limited activity of antifungals against the grains produced by this fungus. To confront these challenges a systematic approach involving the screening of a dozen of essential oils (EOs) of a taxonomically diverse medicinal plants belonging to six botanical families including Burseraceae, Cyperaceae, Euphorbiaceae, Lamiaceae, Myrtaceae, and Poaceae (Table 1) coupled with hyphenated analysis by GC/MS and chemometric analysis was attempted.

Table 1.. The MIC50 of EOs against M. mycetomatis

\begin{tabular}{l|c|}
\multicolumn{1}{c|}{ Plant sources of EOs } & $\begin{array}{c}\text { MIC } \\
(\% \mathbf{v} / \mathbf{v})\end{array}$ \\
\hline Boswellia papyrifera (Delile ex Caill.) Hochst. & 0.0625 \\
\hline Croton zambesicus Müll. Arg. & 0.0625 \\
\hline Cummium cyminum L. & 0.125 \\
\hline Cymbopogon nervatus (Hochst.) Chiov. & 0.0625 \\
\hline Cymbopogon proximus (Hochst. ex A. Rich.) Stapf. & 0.0625 \\
\hline Cyperus rotundus L. & 0.125 \\
\hline Eucalyptus camaldulensis Dehn. & 0.125 \\
\hline Mentha spicata L. & 0.0625 \\
\hline Ocimum bacilicum L. & 0.25 \\
\hline Xylopia aethiopica (Dunal) A. Rich. & 0.0625 \\
\hline Itraconazole (ITZ) & $0.025 \mathrm{ug} / \mathrm{ml}$
\end{tabular}

\section{Methods}

Dried taxonomically identified plants (200 gram) of different morphological parts were subjected to hydrodistillation and their EOs were analyzed by gas chromatography/mass spectrometry (GC-MS)-based metabolomic. Each of these EOs was screened for their in vitro antifungal activity against ten $M$. mycetomatis strains employing our newly developed resazurin assay $^{2}$ whiles, itraconazole was used as positive control. A 10 EOs (descriptor by abbreviation) of detected most abundant 168 compounds (descriptor by experimental RI) .A two-way matrix was constructed comprised $168 \times 10$ points (metabolites $\mathrm{x}$ samples) using the experimental retention index as descriptor for each selected compound. Principal Component Analysis (PCA) and Orthogonal Partial Least Squares - Discriminant Analysis (OPLS-DA) models were implemented on the two-way array after corresponding above-mentioned pre-treatment. In addition, a single- $Y$ OPLS model using antimycetomal as $Y$ variable was also constructed. Results were presented as score plots, loadings lines and $S$-lines. All data analyses were performed using SIMCA 13 software (Umetrics Inc.) on a PC (Intel Xeon processor (32 cores); 32 GB RAM; running on Microsoft Windows 7).

\section{Results}

Ten out of 12 tested EOs exhibited antifungal activity against $M$. mycetomatis isolates at MIC range $(0.125-0.0625 \% \mathrm{v} / \mathrm{v})$. GC/MS analysis resulted in the identification of diverse monoterpenes and sesquiterpenes associated with antifungal activity. Chemometric analysis revealed that the EOs of Commiphora myrrh, Croton zambesicus, Cymbopogon citratus, and Commiphora erythraea exhibited the lowest MIC50 values when tested on the isolates of M. mycetomatis, whilst the EOs of Melaleuca alternifolia, Eucalyptus camaldulensis, and Mentha spicata showed the highest MIC50 values. Other EOs showed moderate antifungal activity in tested isolates. Chemometric analysis revealed that the most active essential oil, $C$. zambezicus, was clearly separated by chemical data as well as $X$. aethiopica and $B$. papyrifera. The S-plot (Figure 1 $\& 2$ ) exhibited the correlating metabolites comprising terpinen-4-ol, $n$-octyl acetate, $p$-cymene, 1,8-cineole, a-terpineol, and linalool indicating these compounds could be considered as candidates for further antimycetomal studies.
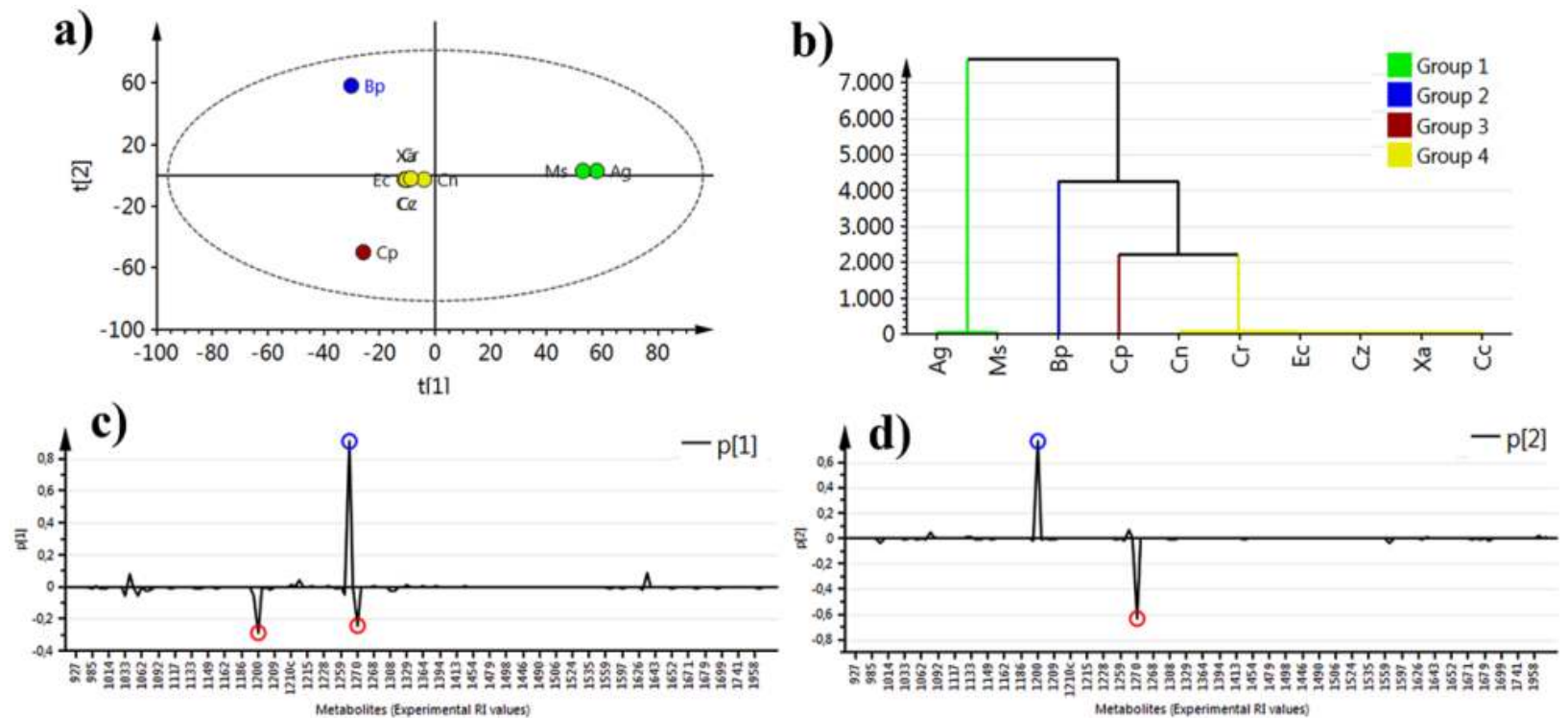

Figure 1. a) PCA S-Plot (PC1 vs $\left.P C 2, R^{2}=0.671\right)$. b) HCA-derived dendrogram. c) PCA-derived Loadings line over PC1. d) PCAderived Loadings line over PC1. Red and blue marks on Loadings lines indicate the metabolites with the strongest contribution on the PC1 or PC2, respectively.

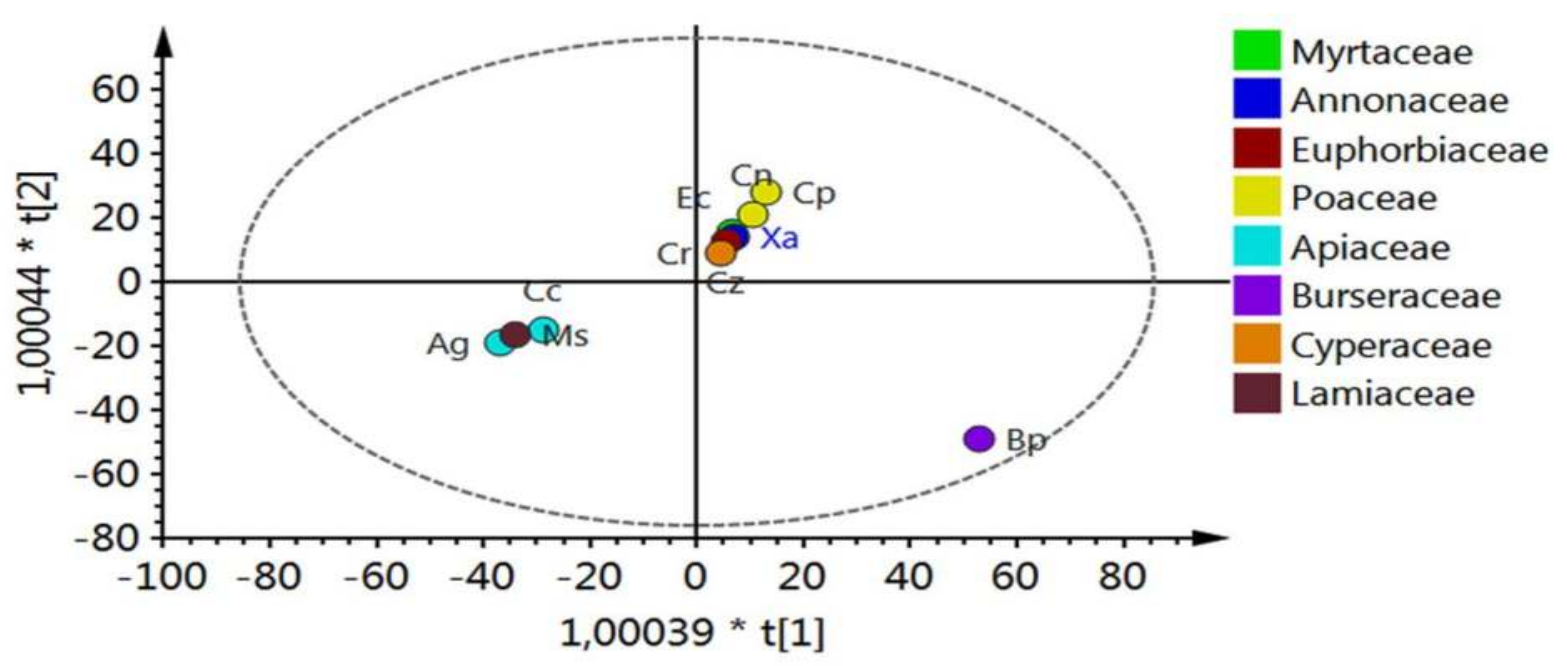

Figure 2. OPLS-DA-derived score plot (PC1 vs $P C 2, R 2=0.805)$ through OPLS model $2+2+0$ and using botanical family as class.

\section{Conclusion}

Chemometric analysis of the EOs chemical constituents coupled with the antimycetomal activity revealed that the following monoterpenes, terpinen-4-ol, $n$-octyl acetate, $p$-cymene, 1,8cineole, $\alpha$-terpineol, and linalool could be considered associated with the antimycetomal activity.

\section{References}

1. van de Sande WWJ et al, PLoS Negl Trop Dis 8(3): e2667. 20142.

2. Khalid SA. $2^{\text {nd }}$ ResNet NPND workshop on natural products against neglected diseases, Nov. 25-28th, 2014, Rio de Janeiro, Brazil. 